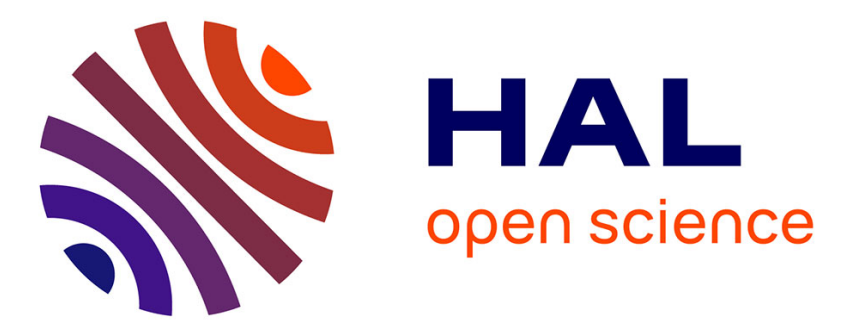

\title{
Determinants of daily pain trajectories and relationship with pain acceptability in hip and knee osteoarthritis. A national prospective cohort study on 886 patients
} Anne-Priscille Trouvin, Marc Marty, Philippe Goupille, Serge Perrot

\section{- To cite this version:}

Anne-Priscille Trouvin, Marc Marty, Philippe Goupille, Serge Perrot. Determinants of daily pain trajectories and relationship with pain acceptability in hip and knee osteoarthritis. A national prospective cohort study on 886 patients. Joint Bone Spine, 2019, 86, pp.245 - 250. 10.1016/j.jbspin.2018.06.009 . hal-03486428

\author{
HAL Id: hal-03486428 \\ https://hal.science/hal-03486428
}

Submitted on 20 Dec 2021

HAL is a multi-disciplinary open access archive for the deposit and dissemination of scientific research documents, whether they are published or not. The documents may come from teaching and research institutions in France or abroad, or from public or private research centers.
L'archive ouverte pluridisciplinaire HAL, est destinée au dépôt et à la diffusion de documents scientifiques de niveau recherche, publiés ou non, émanant des établissements d'enseignement et de recherche français ou étrangers, des laboratoires publics ou privés.

\section{(ㄷ)(1) $\$$}

Distributed under a Creative Commons Attribution - NonCommerciall 4.0 International 


\title{
Determinants of daily pain trajectories and relationship with pain acceptability in hip and knee osteoarthritis. A national prospective cohort study on 886 patients.
}

\author{
Anne-Priscille Trouvin ${ }^{a}$, Marc Marty ${ }^{b, c}$, Philippe Goupille $^{\mathrm{d}, \mathrm{e}}$, Serge Perrot ${ }^{\mathrm{a}}$ \\ a. INSERM U 987, Centre d'Evaluation et Traitement de la Douleur, Hôpital Cochin ; Université Paris Descartes ; 75014 Paris, France \\ b. Service de Rhumatologie, Centre hospitalier Henri Mondor, 94010 Créteil, France \\ c Université Paris XII, 94010 Créteil, France \\ d. CHRU de Tours, Service de Rhumatologie ; 37044 Tours cedex, France \\ e Université de Tours, 37 Tours, France
}

\section{Corresponding author}

Anne-Priscille Trouvin,

Centre d'Evaluation et Traitement de la Douleur, Hôpital Cochin

27 rue du Faubourg Saint Jacques

75014 Paris, France.

Telephone : +33158412559

e-mail: annepriscille.trouvin@aphp.fr 


\section{ABSTRACT}

Objectives: To study daily pain trajectories (DPT) in patients with knee (KOA) and hip osteoarthritis (HOA) over a one-month period and identify relationships with patients characteristics and acceptability.

Methods: This prospective, multicenter cohort study was conducted in France by 602 GPs, on outpatients, with painful KOA or HOA. Patients were asked to fill-in a 28-days daily pain diary. DPT were determined by the difference between daily pain and mean pain over 28 days. Pain peaks were defined as an increase of more than 1 point above the mean for up to three consecutive days. The number of pain peaks over the 28 day period allowed classifying the patient's pain trajectory as either "stable" or "unstable". A logistic regression model was used to identify predicting factors associated with stable pain profile.

Results: Overall, 1645 patients were included and 886 were analysed, (56\% women, 67.8years, BMI $27.6 \mathrm{~kg} / \mathrm{m}^{2}$, pain 6.0, KOA 71.3\%). At one month, stable DPT was found in $59.5 \%$ of the patients whatever OA location. In HOA, a shorter duration of disease and pain, a greater disability and in KOA, a more recent disease, morning stiffness $\geq 15$ minutes and flare up were independent factors associated with "stable" DPT. At one month, acceptable pain state was more frequent $(65.4 \%)$ in patients with stable profiles.

Conclusion: In lower limb OA, pain is mostly stable over a 28-days period. Pain is better accepted when stable, with different determining factors according location. DPT should be considered when establishing HOA and KOA management.

Keywords: osteoarthritis ; pain trajectory ; daily pain ; pain acceptability 


\section{Introduction}

Osteoarthritis (OA) is the most frequent joint disease, leading to pain and disability [1]. Although OA is considered a chronic condition, symptoms are not stable and many patients experience pain fluctuations, e.g. intermittent flare-ups onset during which pain and functional impairment may worsen [2]. In hip (HOA) and knee OA (KOA), pain is the main symptom, is not a stable condition, frequently influenced by exercice and activities [3]. The etiology and impact of the pain fluctuations from OA are not well understood: despite many progresses in the knowledge of OA pain [4], many studies have looked at pain trajectories over two or more years, and functional limitations over a few years [5-9] but very few data are available on the course of pain in KOA and HOA patients on a daily basis [10-16]. Several studies have investigated relationship between pain fluctuations and imaging findings [17] and found that bone marrow lesions and synovial changes were the most important predictor of pain flares [18]. Few studies have investigated intensity of pain fluctuations [14, 16] and tried to define osteoarthritis flare-up [19]. Frequency and duration of flare ups are variables among patients and may define a priori three types of profiles of pain : a slow evolution "stable" profile with steady pain and no apparent flare ups; a "relatively stable" profile with few flare-ups and an "unstable" profile with many flare-ups.

Beside analysis of determinants of pain trajectories, it may be of interest to analyse patient impact of pain, according to the type of pain profile. In OA, there is an increasing interest in Patients Reported Outcome (PROs). The Patient Acceptable Symptom State (PASS), defined as the symptom score level beyond which patients consider themselves well, reflects the concept of "feeling well" [20, 21]. It has been shown that acceptability of pain is associated with intensity of pain [22], but it has never been investigated if pain trajectories may influence

pain acceptability. Because pain is always linked to disability, we also aimed to investigate if pain trajectories were associated with specific disability profile.

The objectives of this observational, prospective, longitudinal cohort study were (i) to describe the frequency of different daily pain trajectories (DPT) in patient suffering from HOA or KOA over 28 days, (ii) to characterize demographic factors, type of pain and disabilities associated with the different defined DPT and (iii) to study the relationship between DPT and acceptability of pain.

This study was designed to allow a better description of DPT related to HOA and KOA and provide a step forward in direction of personalized care, in particular pain relievers. 


\section{Methods}

This observational, prospective, longitudinal cohort study was conducted by 602 French GP during ten months, according to French Ethical Regulations. The protocol was approved by the French «Comité consultatif sur le traitement de l'information en matière de recherche dans le domaine de la santé » and the "Commission Nationale Informatique et Libertê" (CNIL). Patients had to give their written informed consent before inclusion.

\subsection{Study population}

To be recruited, outpatients were to be aged more than 50, to suffer from KOA or HOA according to clinical and radiological criteria and/or clinical criteria of the American College of Rheumatology [23], with an OA pain intensity over the previous 24 hours greater than 3 using a 0 to 10 Numerical Rating Scale (NRS), [24], to need an analgesic treatment for at least one week and be able to fullfill a diary. In case of bilateral OA, the most painful side was considered. Patients were excluded if they have received an injection in the target joint within one month before inclusion, suffered of another disease likely to interfere with the assessment of OA, suffered from serious disease, had symptomatic homolateral HOA and KOA, had any painful joint prosthesis or any symptom suggesting specific disease of the target joint (infection, rheumatoid arthritis...).

\subsection{Assessment}

\subsubsection{At baseline on D0:}

Investigators recorded demographic data (age, gender and social class), and characteristics of the disease (location, duration of the disease and of the current painful episode). The night time and day time pain intensities during the previous day, pain at rest and on movement were measured with a 0 to 10 NRS.

Disability was assessed with the WOMAC index (Western Ontario and McMaster University Osteo-arthritis Index) a 3 domains scale of 24 items, each of which is assessed using a 100 mm visual analogue scale (pain: 5 items, stiffness: 2 items and function: 17 items) [25]. The concept of "patient priorities related to disability", derived from the McMaster-Toronto 
Arthritis Preference disability Questionnaire (MACTAR) [26] was also used. This concept defines the activities the patients considered the most important they want to be improved. Five domains of activities have been determined from the MACTAR concept: home activities, sport activities, outdoor activities, social activities, leisure activities. We asked our patient to choose, in this selection, the main activity they wanted to improve.

The presence of a neuropathic component of the OA pain asserted- by a score higher than 3 using the 10 binary item DN4 questionnaire was used [27]. KOA or HOA joint morning stiffness (less or more than 15 minutes) was recorded. Presence of KOA flare-ups was assessed by means of the validated KOFUS (Knee Osteoarthritis Flare-Ups Score) [19], a 14 items questionnaire with a total score ranging from 0 (no flare up) to 14 , a total score greater than 7 signaling the presence of a flare-up.

To assess the pain PASS threshold, patients were asked with the following question: "Thinking that pain over the preceding 24 hours was only related to your osteoarthritis, and should you stay in this state for the future years, would you consider it as "acceptable" or "unacceptable" [22].

Patients' anxiety-depression state was assessed with a 0 to 10 NRS.

\subsubsection{Every day from D1 to D28,}

Patients had to record the following information in their diary: pain intensity during the previous night (measured with 0 to $10 \mathrm{NRS}$ ) and joint morning stiffness every morning (< or $\geq 15$ minutes) and daytime pain, pain at rest and at movement (measured with 0 to $10 \mathrm{NRS}$ ), daily activities level (less than usual, as usual, more the usual) and treatments taken (drugs and non pharmacological treatment) every evening.

At D7 and D28 acceptability (PAAS) of pain was assessed again. The improvement of pain was estimated by the Minimal Clinically Important Improvement (MCII). To assess the MCII the patients answered to the following question: "Assuming that your pain over the preceding 24 hours was only related to your OA and compared to your pain at baseline, would you say that your pain improved, is unchanged or worsened. If your pain improved please specify whether the improvement is very important, important, fairly important, slightly important, or not at all important ».

In addition, at D28, patients were asked to assess their pain profile on the whole using a 3 level Likert scale: stable, unstable with few peaks, unstable with many peaks. 


\subsection{Interventions}

The treating physicians were free to choose the nature, dosage and duration of the treatments prescribed.

\subsection{Statistical analyses}

\subsubsection{General statistics}

All analyses were performed on Per Protocol population. Patients with major protocol deviations such as inconsistent dates in the patient diary, missing pain intensity assessment for more than 3 consecutive days, missing questionnaire or missing patient diary, inclusion pain intensity at rest or while moving under $3 / 10$, localization of OA missing were excluded from analyses. Baseline characteristics of patients not fulfilling requirement to be kept in the analysis were compared from those of evaluable patients.

Baseline characteristics were described by location of OA and by DPT. The frequencies of profiles and the corresponding two sided $95 \%$ confidence interval $(95 \% \mathrm{CI})$ were determined using the normal approximation. Patients were classified according to their DPT. WOMAC scores were standardized in order to obtain scores ranging from 0 to 10.

\subsubsection{Determination of Daily Pain Trajectory.}

Analyzes were carried out without missing data handling on all patients complying with all inclusion criteria and who completed their diary fully. Daily pain trajectories were assessed by difference between each day pain intensity and the overall mean of pain intensity over 28 days. Peaks of pain were defined by transitory increases of pain intensity of more than 1 point above the mean of all pain intensities over the 28 day follow-up period for three consecutive days or less (Figure 1). The daily pain trajectory of each patient was determined using a method based on the distribution of the number of peaks of pain during the 28 day follow-up period in the whole survey sample. If for a given patient this number was less than or equal to the $25^{\text {th }}$ percentile of the distribution the trajectory was classified "stable". If it was between the $25^{\text {th }}$ percentile and the median value excluded, the evolution was considered as "stable with few peaks". It was classified as "unstable" if the number of pain peaks was equal to or greater than the median of the sample. The relevance of the method was validated by a 
planned interim analysis performed on the data of the first 100 patients.

2.4.3. Identification of factors associated with Daily Pain Trajectory.

Independent factors associated with DPT were determined by means of a descending logistic regression with a stay level of 5\%, selecting potential explanatory variables from the set of variables significant at the 5\% level when comparing the characteristics of patients with stable or stable with few peaks profiles on one hand and those with unstable profile on the other using $\mathrm{Chi}^{2}$ or Fisher Exact tests for categorical data, a one way analysis of variance (ANOVA) for quantitative data.

\subsubsection{Others analyses}

The evolution profile determined based on pain intensity NRS over the 28-day follow-up period was compared to the patients' self-assessment at D28 using Cohen's Kappa coefficient.

Pain PASS threshold was defined as the $75^{\text {th }}$ percentile of mean pain intensity of night and day of the last day of patients considering their pain acceptable. Patients with a pain value under this threshold were considered with a pain acceptable state and those with a greater value were considered with a pain unacceptable state (this value was calculated at D28) [22].

Pain MCII threshold was defined as the $75^{\text {th }}$ percentile of the mean of the difference of night and day pain intensity between D0 and D28 for patients considering a minimal "fairly important" improvement between D0 and 28 [28].

Statistical analysis was performed using SAS® version 9.1 for Windows (SAS Institute Inc., Cary, NC, USA).

\subsection{Sample size}

No confirmed hypotheses allowed a calcualtion on a primary end points.

\subsection{Role of the funding source}

This study has been developed with an unrestricted grant from SANOFI 


\section{Results}

\subsection{Disposition of patients}

The survey was proposed by mailing to a sample of 10000 French GPs of whom 1066 agreed to participate.Among these 602 GPs (men $83.0 \%$, mean \pm SD age $52.9 \pm 7.3$ years) recruited 1645 patients propectively over 10 consecutive months. A total of 759 observations were excluded from the analyses because of major protocol deviations; mostly inconsistent dates in the patient diary $(n=671)$, missing pain intensity assessment for more than 3 consecutive days $(n=488)$, missing questionnaire or missing patient diary $(n=312)$, neither pain intensity at rest nor pain while moving inclusion greater than or equal to $3 / 10(n=80)$, localization of OA missing $(n=24)$. Baseline characteristics of patients not fulfilling requirement to be kept in the analysis did not differ from those of evaluable patients. No clinical differences were obseerved between HOA and KOA.

\subsection{Patients at inclusion}

Most of the 886 evaluable patients were women (56.0\%) with mean \pm SD age $67.8 \pm 9.3$ years, and a BMI of $27.6 \pm 4.6 \mathrm{~kg} / \mathrm{m}^{2}$. A majority of them $(632 ; 71.3 \%)$ suffered from $\mathrm{KOA}$

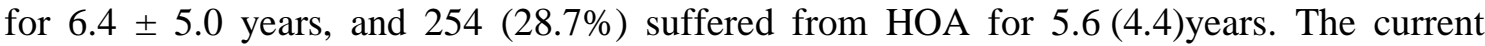
painful episode lasted for $15.5 \pm 15.0$ days, with mean pain intensity of $6.0 \pm 1.5$ during daytime and 6.7 (1.5) while moving. All types of activities (at home, outdoor, sports, leisure time, and social activities) were affected for a large majority of patients. KOA flare-ups (KOFUS $\geq 7 / 14$ ) were observed for $62.7 \%$ of the patients with KOA at baseline. The scores of 3 domains of the standardized WOMAC scale were 5.3 (1.5) for pain, $5.5 \pm 1.7$ for stiffness and $5.3 \pm 1.7$ for function (Table 1).

\subsection{Daily Pain Trajectories during a one-month study period}

The $25^{\text {th }}$ percentile and the median of the distribution of the number of peaks of pain were equal; therefore only the "stable" and "unstable" profiles of pain evolution could be identified (Figure 2). For 527 patients $(59.5 \%$ (95\% CI 56.2\%; 62.7\%) the pain trajectory was 
considered as "stable" during the 28-day follow up period. It was "unstable" for 359 patients (40.5\% (95\% CI 37.3\%; 43.8\%). The results were very similar for patients with HOA and KOA (59.5\%) (Table 2 and Figure 2).

\subsection{Correlation between patient's perception and daily pain trajectory}

Patients' self assessment (stable pain or unstable pain) of pain trajectory was poorly correlated with pain trajectory determined by pain intensity NRS over the 28 days with a Cohen Kappa coefficient at 0.14 for the overall population ( 0.13 for KOA and 0.16 for HOA).

\subsection{Independent factors associated with Daily Pain Trajectory}

Different sets of independent factors associated with the "stable" pain trajectory were found for hip and knee OA. Baseline characteristics of patients according to Daily Pain Trajectories were displayed in Table 3.

In HOA patients, the odds of "stable" trajectories were 1.416 higher when the duration of the disease decreased by 5 years $(\mathrm{p}<0.05)$ and 1.912 higher when the duration of the current episode decreased by 1 month $(\mathrm{p}<0.05)$. An increase of one point of the WOMAC score increased the odds of "stable" profiles by $1.186(\mathrm{p}<0.05)$.

In KOA patients, a 5 year shorter disease evolution increased the odds of "stable" trajectory by $1.364(\mathrm{p}<0.05)$. The odds of "stable" trajectory were 1.584 times higher when the duration of morning stiffness was less than or equal to 15 minutes. Similarly the odds of "stable" trajectory were 1.755 times higher for patients with a KOFUS greater than or equal to 7. Age, gender, BMI, professional activities, anxiety, depression, pain intensity, joint stiffness, function assessed by WOMAC, neuropathic character of pain were not associated with profile of pain (Table 4).

\subsection{Patient Acceptability Stable State (PASS) and correlation with DPT}

At D28, value of pain intensity corresponding to the PASS was $2.5 / 10$ and $58.5 \%(517 / 884)$ of patients were classified as reaching a PASS for pain. Acceptable pain state was more 
frequent for patients with "stable" trajectories $(65.4 \%(344 / 526))$ than for those with "unstable" trajectories (48.3\% (173/358) with p value<0.0001 [Appendix A, Table S1; See the supplementary material associated with this article online]. Baseline characteristics of patients according to PASS were displayed in Appendix A, Table S2.

\subsection{Minimal Clinically Important Improvement (MCII)}

At D28, value of pain intensity difference of the MCII (between D0 and D28) was 1.5/10, with $77 \%(681 / 886)$ of the patients considering their pain as significantly improved at the end of the study period. A MCII value was more frequently reached for patients on "stable" trajectories $(81.7 \%(430 / 527))$ than for those with "unstable" trajectories $(70.1 \%(251 / 359))$ with $\mathrm{p}$ value $<0.0001$.

\section{Discussion}

This observational, prospective, longitudinal cohort study aimed to determine the types and frequencies of different daily pain trajectories in patient suffering from HOA or KOA and to identify independent factors associated with the different trajectories. In addition, relationships between daily pain trajectories, pain acceptability, and patients disability priorities were studied.

Only two profiles of pain evolution were identified, the stable trajectory being more frequent $(59,5 \%)$. Our a priori definition of "stable", "relatively stable (with few peaks)" and unstable trajectories was based on the distribution of the number of peaks of pain for the study population. Since the distribution was very asymmetrical, with a common value for the $25^{\text {th }}$ percentile and the median, no patient had a stable trajectory with few peaks. Our study demonstrates that OA pain is a relatively stable condition, over a period of one month, both in HOA and KOA. In 59.5\% of the patients, pain trajectory was considered as "stable" during the 28-day follow up period. It was considered as "unstable" in $40.5 \%$ of them. The results were very similar for patients with $\mathrm{HOA}(59.4 \%$ stable) and KOA (59.5\% stable). Identifying patients trajectory will clearly help pain relievers' prescription, patients with "unstable" require rescue analgesic treatment to be anticipated. 
Our results are close to that of a longitudinal study on weekly fluctuations showing that $51 \%$ of patients had a fluctuation of less than 2 points on the WOMAC pain subscore on a weekly analysis, thus considered stable [14]. In a study in patients awaiting surgery, Kapstad and coworkers [29] demonstrated that HOA patients were relatively stable, while patients with KOA demonstrated significant deterioration, especially in female patients. This reflects probably a very different situation, patients awaiting surgery being at a severe stage of the disease, and in a specific awaiting situation. In fact, correlation between patients' fluctuations self report and pain fluctuations from the daily diary was poor in our study (kappa coefficient at 0.14 ). Meaningful changes of pain intensity should be probably analyzed in term of pain impact and pain coping [30] but also in the direction of pain changes, i.e. improvement or worsening. In our study we demonstrated that MCII was more frequently reached in patients with stable condition: this may suggest that improvement of pain may be confounded by the patients as a stable condition, although unstable pain may more frequently reflect worsening of the pain. This is corroborated by the fact that pain acceptability was more frequent in stable condition.

In univariate analyses, several factors have been found statistically significant different in patients with stable pain trajectory vs unstable pain trajectory: disease duration, daytime pain intensity, pain at rest, duration of morning stiffness, WOMAC pain subscore, WOMAC stiffness subscore. Parry et al. found the same factors predicting significant pain variability (higher WOMAC Pain score, longer time since onset and morning stiffness) in a cohort of more than 700 patients [31]. Interestingly, BMI, sex, presence of neuropathic component, patients' disability priorities, mood disorders (anxiety and depression levels), were not associated with a specific daily pain trajectory. These findings are similar to those of Schneider and coworkers, in 194 patients with osteoarthritis of the lower limb, mean pain intensity was collected over a week, no significant effect of psychological factors were found in predicting within-person variability in pain. Studied psychological factors included Beck Depression Inventory, Arthritis Impact Measurement Scale tension subscale for anxiety, Arthritis Self-Efficacy Scale and Coping Strategies Questionnaire with catastrophizing subscale. [16].

Multivariate analyses have demonstrated different independent factors associated with stable pain trajectory in $\mathrm{HOA}$ and $\mathrm{KOA}$, demonstrating that these $2 \mathrm{OA}$ locations are different clinical conditions. For HOA, factors independently associated with a stable trajectory were a shorter duration of disease, a shorter duration of the painful episode, a greater disability and 
for KOA a more recent disease, 15 minutes or less of morning stiffness and presence of flare ups.

For KOA, the main parameter associated with stable pain trajectory was the presence of flare up defined by KOFUS score. This means that patients with KOA flare-ups have often a stable DPT. This could be surprising, it may be because patients with a stable trajectory have a better acceptability of pain and this point highlights the complex relationships between factors as Daily Pain Trajectories, Flare-ups and acceptability of pain (Appendix A, Table S3).

Our study has some limitations. The 28 day follow-up period may be considered too short to really assess stability of pain in OA patients. We consider that a period of 28 days was enough to assess daily profile of pain [30]. The definition of peaks and trajectories of pain were selected by the authors. And even if the relevance of the method was validated by a planned interim analysis performed on the data of the first 100 patients, this can be a source of bias. The fact that patients' self assessment (stable pain or unstable pain) of pain trajectory was poorly correlated with pain trajectory determined by pain intensity NRS over the 28 days could be an argument for bias, but in absence of gold standard of definition for stable or unstable profile, the direction of bias can not be known.

We anticipated a much higher frequency for peaks of pain which would have allowed defining an intermediate profile of stable pain with few peaks. Further investigations may explore less restrictive definitions of peaks and other definitions of pain trajectories. Another possible approach could be to sum up peak- trough values over the follow up period.

Another major concern is the high number of observations we had to exclude from the analyses. Given our definitions of peaks of pain, we needed exhaustive patient diary data over the 28 day follow-up period. In our situation, missing data cannot be considered missing at random, as, for instance, patients with low level of pain may be more likely to forget to fill-in their diary. Similarly, patients who are not relieved by their treatment may also be more likely to forget their daily filling-in. This leads to biased results for our primary outcome criterion. However, a comparison of the baseline characteristics of included and analyzed patients showed no significant difference for any variable

In conclusion, this prospective study reports on fully daily diary data during a one-month study period of 886 patients without any extrapolated data give a precise feature of OA pain trajectory. This study demonstrates that OA pain intensity is relatively stable within 1 month. Pain fluctuations do not differ between HOA and KOA, are increased with disease duration. Pain intensity stability has few independent determining factors, different between HOA and KOA, especially on function, but do not depends on mood disturbances, neuropathic pain 
component, sex nor patients' priorities. Our study also demonstrates that pain trajectories have a direct impact on pain acceptability. These data should be integrated for a better individualized management of $\mathrm{OA}$ pain on a daily practice basis and help identifying "unstable" patients who might require a more tailored pain relievers prescription.

\section{Appendix A. Supplementary data}

Supplementary data (Tablesd S1-S3) associated with this article can be found in the online version at ...

\section{Acknowledgments:}

This paper has been prepared with the help of a medical writer, Dr Christian Kempf, from CEGEDIM.

\section{Conflict of interest disclosures :}

MM and SP have received fees from SANOFI for advisory.

APT and PG: no conflict of interest in relation with this study. 


\section{References}

[1] Woolf AD, Pfleger B. Burden of major musculoskeletal conditions. Bull World Health Organ 2003;81:646-56.

[2] Dieppe PA. Relationship between symptoms and structural change in ostoarthritis. What are important targets for osteoarthritis therapy. J Rheumatol 2004;31:supp 70;50-3.

[3] Peat G, McCarney R, Croft P. Knee pain and osteoarthritis in older adults: a review of community burden and current use of primary health care. Ann Rheum Dis 2001;60:91-7.

[4] Trouvin AP, Perrot S. Pain in osteoarthritis. Implications for optimal management. Joint Bone Spine 2017 Sep 6. pii: S1297-319X(17)30156-2. doi: 10.1016/j.jbspin.2017.08.002.

[5] Bastick AN, Verkleij SP, Damen J, et al. Defining hip pain trajectories in early symptomatic hip osteoarthritis--5 year results from a nationwide prospective cohort study (CHECK). Osteoarthritis Cartilage 2016;24:768-75.

[6] Bastick AN, Wesseling J, Damen J, et al. Defining knee pain trajectories in early symptomatic knee osteoarthritis in primary care: 5-year results from a nationwide prospective cohort study (CHECK). Br J Gen Pract 2016;66:e32-9.

[7] Nicholls E, Thomas E, van der Windt DA, Croft PR, Peat G. Pain trajectory groups in persons with, or at high risk of, knee osteoarthritis: findings from the Knee Clinical Assessment Study and the Osteoarthritis Initiative. Osteoarthritis Cartilage 2014;22:2041-50.

[8] Verkleij SP, Hoekstra T, Rozendaal RM, et al. Defining discriminative pain trajectories in hip osteoarthritis over a 2-year time period. Ann Rheum Dis 2012;71:1517-23.

[9] Helminen EE, Sinikallio SH, Valjakka AL, Väisänen-Rouvali RH, Arokoski JP. Determinants of pain and functioning in knee osteoarthritis: a one-year prospective study. Clin Rehabil. 2016;30:890-900

[10] Van Dijk GM, Dekker J, Veenhof C, vanden Ende CH, Carpa Study Group. Course of functionnal status and pain in osteoarthritis of the hip or knee: a systematic review of the literature. Arthritis Rheum 2006;55:779-85.

[11] Hawker GA, Stewart L, French MR, et al. Understanding the pain experience in hip and knee osteoarthritis - an OARSI/OMERACT initiative. Osteoarthritis Cartilage 2008;16: 415-422.

[12] Gooberman-Hill R.D, Wollhead G, MacKichan F, Ayis S, Williams S, Dieppe P. Assessing chronic joint pain: lessons form a focus group study. Arthritis Rheum 2007;57:666-671.

[13] Wollhead G, Gooberman-Hill R, Dieppe P, Hawker G. Night pain in hip and knee osteoarthritis : a focus group study. Arthritis Care Research 2010;7:944-949.

[14] Hutchings A, Calloway M, Choy E, et al. The Longitudinal Examination of Arthritis Pain (LEAP) Study: relationships between weekly fluctuations in patient-related joint pain and other health outcomes. J Rheumatol 2007;4:2291-300.

[15] Perrot S, Rozenberg S, Moyse D, Legout V, Marty M. ; cercle d'étude de douleur en rhumatologie (CEDR). Comparison of daily, weekly or monthly pain assessments in hip and knee osteoarthritis. A 29-day prospective study. Joint Bone Spine 2011;78:510-5. 
[16] Schneider S, Junghaenel DU, Keefe FJ, Schwartz JE, Stone AA, Broderick JE. Individual differences in the day-to-day variability of pain, fatigue, and well-being in patients with rheumatic disease: associations with psychological variables. Pain 2012;153:813-22.

[17] Zhang Y, Nevitt M, Niu Jet al. Fluctuation of knee pain and changes in bone marrow lesions, effusions, and synovitis on magnetic resonance imaging. Arthritis Rheum 2011;63:691-9.

[18] Torres L, Dunlop DD, Peterfy C, et al. The relationship between specific tissue lesions and pain severity in persons with knee osteoarthritis. Osteoarthritis Cartilage 2006;14:1033-40.

[19] Marty M, Hilliquin P, Rozenberg S, et al. Validation of the KOFUS (Knee Osteoarthritis Flare-ups Score). Jone Bone Spine 2009;76:268-272.

[20] Dougados M, Moore A, Yu S, Gitton X. Evaluation of the patient acceptable symptom state in a pooled analysis of two multicentre, randomised, double-blind, placebo-controlled studies evaluating lumiracoxib and celecoxib in patients with osteoarthritis. Arthritis Res Ther 2007;9:R11.

[21] Kvien TK, Heiberg T, Hagen KB. Minimal clinically important improvement/difference (MCII/MCID) and patient acceptable symptom state (PASS): what do these concept mean? Ann Rheum Dis 2007;66:40-1.

[22] Tubach F, Ravaud P, Baron G, et al. Evaluation of clinically relevant states in patient reported outcomes in knee and hip osteoarthritis: the patient acceptable symptom state. Ann Rheum Dis 2005;64:34-7.

[23] Altman R, Asch E, Bloch D, et al. Development of criteria for the classification and reporting of osteoarthritis. Classification of osteoarthritis of the knee. Diagnostic and Therapeutic Criteria Committee of the American Rheumatism Association. Arthritis Rheum 1986;29:1039-1049.

[24] McCaffery, JA. Psychologically coping: pain that won't go away. COMSIG Rev 1993;2:71-3.

[25] Bellamy N, Buchanan WW, Golddsmith CH, Campbell J, Stitt WL. Validation of WOMAC : a health status onstrument for measuring clinically important patient relevant outcomes to antirheumatic drig therapy in patients with osteoarthritis og the hip or knee. J Rheumatol 1988;15:1833-40.

[26] Tugwell P, Bombardier C, Buchanan WW, Goldsmith CH, Grace E, Hanna B. The MACTAR Patient Preference Disability Questionnaire - An individualized functional priority approach for assessing improvement in physical disability in clinical trials in rheumatoid arthritis. J Rheumatol 1987;14:446-51.

[27] Bouhassira D, Attal N, Alchaar H, et al. Comparison of pain syndromes associated with nervous or somatic lesions and development of a new neuropathic pain diagnostic questionnaire (DN4). Pain 2005;114:29-36.

[28] Tubach F, Ravaud P, Baron G, et al. Evaluation of clinically relevant changes in patient reported outcomes in knee and hip osteoarthritis: the minimal clinically important improvement. Ann Rheum Dis 2005;64:29-33.

[29] Kapstad H, Rustøen T, Hanestad BR, Moum T, Langeland N, Stavem K. Changes in pain, stiffness and physical function in patients with osteoarthritis waiting for hip or knee joint replacement surgery. Osteoarthritis Cartilage 2007;15:837-43. 
[30] Perrot S, Poiraudeau S, Kabir M, et al. Active or passive pain coping strategies in hip and knee osteoarthritis? Results of a national survey of 4,719 patients in a primary care setting. Arthritis Rheum 2008;59:1555-62.

[31] Parry E, Ogollah R, Peat G. Significant pain variability in persons with, or at high risk of, knee osteoarthritis: preliminary investigation based on secondary analysis of cohort data. BMC Musculoskelet Disord. 2017;18:80 
Table 1 : Patients characteristic at baseline - figures are means (SD) unless stated otherwise (KOA and HOA patients)

\begin{tabular}{|c|c|c|}
\hline & $\begin{array}{c}\text { Hip OA } \\
(\mathrm{N}=254)\end{array}$ & $\begin{array}{l}\text { Knee OA } \\
(\mathrm{N}=632)\end{array}$ \\
\hline Age (years) & $68.4(9.0)$ & $67.5(9.4)$ \\
\hline Female n $(\%)$ & $123(52.2 \%)$ & $364(57.6 \%)$ \\
\hline $\operatorname{BMI}\left(\mathrm{kg} / \mathrm{m}^{2}\right)$ & $27.0(4.3)$ & $27.9(4.6)^{*}$ \\
\hline Duration of the disease (years) & $5.6(4.4)$ & $6.4(5.0)^{*}$ \\
\hline Duration of the current episode (days) & $15.6(13.8)$ & $15.4(15.5)$ \\
\hline Mean pain during the previous night - NRS $0-10$ & $5.0(2.2)$ & $4.9(2.1)$ \\
\hline Mean pain during daytime NRS $0-10$ & $6.1(1.6)$ & $6.0(1.5)$ \\
\hline Pain at rest NRS $0-10$ & $4.3(1.9)$ & $4.4(1.8)$ \\
\hline Pain while moving NRS $0-10$ & $6.8(1.5)$ & $6.7(1.5)$ \\
\hline Duration of morning stiffness $\geq 15$ minutes $\mathrm{n}(\%)$ & $193(76.6 \%)$ & $425(67.4 \%)$ \\
\hline \multicolumn{3}{|l|}{ Most affected activities (MACTAR): } \\
\hline At home & $192(81.0 \%)$ & $453(75.2 \%)$ \\
\hline Outdoor & $233(98.3 \%)$ & $589(97.8 \%)$ \\
\hline Sports & $200(85.8 \%)$ & $514(87.9 \%)$ \\
\hline Leisure time & $227(96.2 \%)$ & $658(94.8 \%)$ \\
\hline Social activities & $114(60.8 \%)$ & $363(60.6 \%$ \\
\hline Depressive state NRS 0-10 & $3.1(2.5)$ & $2.8(2.3)$ \\
\hline Anxiety level NRS 0-10 & $3.3(2.5)$ & $3.1(2.3)$ \\
\hline KOFUS score $\geq 7$ (score ranges from 0 to 14$)$ & - & $371(62.1 \%)$ \\
\hline WOMAC Pain domain (scores ranges 0 to 10$)$ & $5.4(1.6)$ & $5.3(1.5)$ \\
\hline WOMAC Stiffness domain (scores ranges 0 to 10 ) & $5.7(1.7)$ & $5.5(1.7)$ \\
\hline WOMAC Function domain (scores ranges 0 to 10 ) & $5.6(1.7)$ & $5.2(1.6)^{*}$ \\
\hline DN4 score $>4($ score ranges from 0 to 10$)$ & $33(13.1 \%)$ & $85(13.6 \%)$ \\
\hline
\end{tabular}

. BMI: Body Mass Index; NRS: Numerical Rating Scale; MACTAR : McMaster-Toronto Arthritis Preference disability Questionnaire; KOFUS: Knee Osteoarthritis Flare Up Score; WOMAC: Western Ontario and McMaster University Osteo-arthritis Index; DN : neuropathic pain; OA: OsteoArthritis * $\mathrm{p}<0.01$ 
Table 2 : Daily Pain Trajectory from D1 to D28 (KOA and HOA patients)

\begin{tabular}{|l|c|c|c|}
\hline & $\begin{array}{c}\text { Hip OA } \\
(\mathrm{N}=254) \\
\mathrm{n}(\%)\end{array}$ & $\begin{array}{c}\text { Knee OA } \\
(\mathrm{N}=632) \\
\mathrm{n}(\%)\end{array}$ & $\begin{array}{c}\text { Total } \\
(\mathrm{N}=886) \\
\mathrm{n}(\%)\end{array}$ \\
\hline Stable according to pain diary & $151(59.4 \%)$ & $376(59.5 \%)$ & $527(59.5 \%)$ \\
\hline Unstable according to pain diary & $103(40.6 \%)$ & $256(40.5 \%)$ & $359(40.5 \%)$ \\
\hline Stable self report & $60(24.2 \%)$ & $161(26.2 \%)$ & $221(25.6 \%)$ \\
\hline Unstable self report & $188(78.5 \%)$ & $454(73.8 \%)$ & $642(74.4 \%)$ \\
\hline
\end{tabular}


Table 3 : Patients characteristic at baseline according to DPT Figures are means (SD) unless stated otherwise (KOA and HOA patients)

\begin{tabular}{|c|c|c|}
\hline & $\begin{array}{c}\text { Stable } \\
(\mathrm{N}=527)\end{array}$ & $\begin{array}{l}\text { Unstable } \\
(\mathrm{N}=359)\end{array}$ \\
\hline Age (years) & $67.2(9.3)$ & $68.6(9.2)$ \\
\hline Female n (\%) & $296(56.2 \%)$ & $200(55.9 \%)$ \\
\hline $\operatorname{BMI}\left(\mathrm{kg} / \mathrm{m}^{2}\right)$ & $27.6(4.4)$ & $27.6(4.8)$ \\
\hline Duration of the disease (years) & $5.7(4.3)$ & $6.9(5.5)^{* * *}$ \\
\hline Duration of the current episode (days) & $14.6(14.8)$ & $16.7(15.3)$ \\
\hline Mean pain during the previous night - NRS 0-10 & $5.0(2.1)$ & $4.8(2.1)$ \\
\hline Mean pain during daytime NRS $0-10$ & $6.1(1.5)$ & $5.8(1.5)^{* *}$ \\
\hline Pain at rest NRS $0-10$ & $4.6(1.8)$ & $4.1(1.8)^{* * *}$ \\
\hline Pain on movement NRS $0-10$ & $6.7(1.5)$ & $6.6(1.5)$ \\
\hline Duration of morning stiffness $\geq 15$ minutes $\mathrm{n}(\%)$ & $388(73.6 \%)$ & $230(64.6 \%)^{* *}$ \\
\hline \multicolumn{3}{|l|}{ Most affected activities (MACTAR): } \\
\hline At home & $394(77.9 \%)$ & $251(75.4 \%)$ \\
\hline Outdoor & $233(97.8 \%)$ & $327(98.2 \%)$ \\
\hline Sports & $437(88.3 \%)$ & $277(85.8 \%)$ \\
\hline Leisure time & $480(95.0 \%)$ & $315(95.5 \%)$ \\
\hline Social activities & $317(62.6 \%)$ & $190(57.6 \%$ \\
\hline Depressive state NRS 0-10 & $3.0(2.4)$ & $2.7(2.3)$ \\
\hline Anxiety level NRS 0-10 & $3.2(2.4)$ & $3.0(2.3)$ \\
\hline WOMAC Pain domain (scores ranges 0 to 10 ) & $5.4(1.6)$ & $5.2(1.4)^{*}$ \\
\hline WOMAC Stiffness domain (scores ranges 0 to 10 ) & $5.7(1.7)$ & $5.4(1.6)^{*}$ \\
\hline WOMAC Function domain (Scores ranges 0 to 10 ) & $5.4(1.7)$ & $5.2(1.6)$ \\
\hline DN4 score $>4$ (score ranges from 0 to 10$)$ & $64(12.2 \%)$ & $54(15.4 \%)$ \\
\hline
\end{tabular}

BMI: Body Mass Index; NRS: Numerical Rating Scale; MACTAR : McMaster-Toronto Arthritis Preference disability Questionnaire; KOFUS: Knee Osteoarthritis Flare Up Score; WOMAC: Western Ontario and McMaster University Osteoarthritis Index; DN : neuropathic pain; OA: OsteoArthritis. ${ }^{*} \mathrm{p}<0.05 ; * * \mathrm{p}<0.01 ; * * * \mathrm{p}<0.001$ 
Table 4 : Independent factors associated with a stable profile

\begin{tabular}{|c|c|c|}
\hline & OR & $95 \%$ CI \\
\hline \multicolumn{3}{|l|}{ Hip $\mathrm{OA}^{\mathrm{a}}$} \\
\hline Short duration of the disease (OR for five years) & 1.416 & {$[1.035 ; 1.942]^{*}$} \\
\hline Short Duration of the current episode (OR for one month) & 1.921 & {$[1.062 ; 3.639]^{*}$} \\
\hline High disability assessed by WOMAC (OR for one unit) & 1.186 & {$[1.006 ; 1.397]^{*}$} \\
\hline \multicolumn{3}{|l|}{ Knee $\mathrm{OA}^{\mathrm{b}}$} \\
\hline Short Duration of the disease (OR for five years) & 1.357 & $\begin{array}{c}{[1.142 ;} \\
1.618]^{* * *}\end{array}$ \\
\hline Morning stiffness $\geq 15$ minutes $^{\mathrm{c}}$ & 1.584 & {$[1.094 ; 2.294]^{*}$} \\
\hline Presence of flare up - KOFUS $\geq 7^{\mathrm{d}}$ & 1.755 & {$[1.230 ; 2.503]^{* *}$} \\
\hline \multicolumn{3}{|c|}{$\begin{array}{l}\text { a: Descending logistic regression AIC }=309.3 \text { Likelihood ratio test } \mathrm{Chi}^{2}=12.14, \mathrm{p}=0.0069, \mathrm{~N}=233 \text { patients. } \\
\text { b: Descending logistic regression AIC }=765.9 \text { Likelihood ratio test } \mathrm{Chi}^{2}=31.30, \mathrm{p}<10^{-4}, \mathrm{~N}=586 \text { patients } \\
\text { c: vs. morning }<15 \text { minutes. } \\
\text { d: } v s . \text { KOFUS }<7 / 14 \text {. } \\
\text { KOFUS: Knee Osteoarthritis Flare Up Score. } \\
* \mathrm{p}<0.05 ; * * \mathrm{p}<0.01 ; * * * \mathrm{p}<0.001\end{array}$} \\
\hline
\end{tabular}




\section{FIGURES}

\section{Figure 1: Determination of peaks of pain}

Peaks of pain were defined by transitory increases of pain intensity of more than 1 point above the mean of all pain intensities over the 28 day follow-up period for three consecutive days or less. In this example, the mean of all pain measurements of all patients over the 28 day study period was 3.2, leading to a threshold value of 4.2. The patient has 4 periods of time during which the mean daily pain was above the threshold value, but one of these periods lasted 4 days. Thus, this patient presented 3 peaks of pain.

\section{Figure 2: Distribution of peaks of pain}

For both KOA and HOA, 59.5\% of the patients presented no peaks of pain. The median and $25^{\text {th }}$ of the distribution of the number of peak were both equal to 0 . Patients with no peaks of pain were considered to have a stable pain profile, patients with one or more peaks were considered to have unstable profiles. 


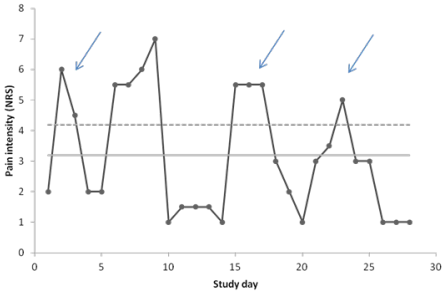

- Mean daily pain _ Overall mean pain _..-- Threshold 


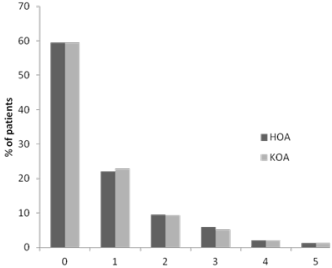

Number of peaks of pain during the 28 day follow up period 